\title{
Effect of Temporal Resolution on the Accuracy of ADCP Measurements
}

\author{
Juan A. González-Castro ${ }^{1}$, Kevin Oberg ${ }^{2}$, James J. Duncker ${ }^{2}$
}

\begin{abstract}
The application of acoustic Doppler current profilers (ADCP's) in river flow measurements is promoting a great deal of progress in hydrometry. ADCP's not only require shorter times to collect data than traditional current meters, but also allow streamflow measurements at sites where the use of conventional meters is either very expensive, unsafe, or simply not possible. Moreover, ADCP's seem to offer a means for collecting flow data with spatial and temporal resolutions that cannot be achieved with traditional current-meters. High-resolution data is essential to characterize the mean flow and turbulence structure of streams, which can in turn lead to a better understanding of the hydrodynamic and transport processes in rivers. However, to properly characterize the mean flow and turbulence intensities of stationary flows in natural turbulent boundary layers, velocities need to be sampled over a long-enough time span. The question then arises, how long should velocities be sampled in the flow field to achieve an adequate temporal resolution? Theoretically, since velocities cannot be sampled over an infinitely long time interval, the error due to finite integration time must be considered. This error can be estimated using the integral time scale. The integral time scale is not only a measure of the time interval over which a fluctuating function is correlated with itself but also a measure of the time span over which the function is dependent on itself. This time scale, however, is not a constant but varies spatially in the flow field. In this paper we present an analysis of the effect of the temporal resolution (sampling time span) on the accuracy of ADCP measurements based on the integral time scale. Single ping velocity profiles collected with frequencies of $1 \mathrm{~Hz}$ in the Chicago River at Columbus Drive using an uplooking $600 \mathrm{kHz}$ ADCP are used in this analysis. The integral time scale at different depths is estimated based on the autocorrelation function of the velocity fluctuations and is used to evaluate the mean-square error as a function of the integration time. The implications of these errors in typical ADCP measurements for discharge estimates in natural streams are discussed.
\end{abstract}

${ }^{1}$ Post-doctoral Research Associate, Illinois Water Resources Center, University of Illinois,

221 N. Broadway, Urbana, IL 61801

${ }^{2}$ Hydrologist, U.S. Geological Survey, 221 N. Broadway, Urbana, IL 61801 


\section{Introduction and objectives}

Acoustic Doppler profilers (ADCP) have been in use for measuring flows in natural and man-made watercourses for over a decade. The effect of the spatial averaging performed by acoustic profilers on the accuracy of flow measurements near solid boundaries (Gordon, 1996) is known to most ADCP users. It has long been recognized that the sampling time also affects the accuracy of mean velocity estimates. However, only qualitative guidelines to select the time span needed to achieve meaningful estimates of the mean velocity based on ADCP measurements have been reported (e.g., Droz et al., 1998, Barua y Rahman, 1998, González et al. 1996). Droz et al. (1998) have estimated that a sampling time of 5 minutes should be used for estimating mean streamwise velocities with an ADCP. Baru and Rahman (1998) reported that to estimate turbulent intensities for flows in the Jamuna River in Bangladesh from broadband ADCP velocity records with a resolution frequency of about $2 \mathrm{~Hz}, 15$-minute averaging was necessary. González et al. (1996), based on fixed broadband ADCP measurements collected at a frequency of $0.25 \mathrm{~Hz}$ in the Chicago River at Romeoville Illinois, reported that records of no less than 5 minutes are necessary to clearly define the streamwise velocity profiles at the center of the channel.

Several researchers have identified the importance of selecting the proper sampling time to achieve accurate estimates of mean velocity and turbulence quantities in the open-channel flows as well as in near-bed tidal boundary layers. McQuivey (1973), after estimating turbulence quantities and mean velocities in the Mississippi River by averaging data collected with a parabolic-shaped hot-film sensor over a time span of $4 \mathrm{~min}$, suggested that this is probably not long enough for a river system of this size. Soulsby (1980) examined the effect of several factors associated with the digitization rate and record length on the accuracy of the measurement of the mean velocity, the variance of the downstream and vertical velocity fluctuations, as well as of their covariance in a near-bed tidal boundary layer. He concluded that the sampling period and digitization rate should be chosen considering the low- and high-frequency spectral cutoff, stationarity, sampling variability, sensor averaging and size of the data set. In particular, he reported theoretical expressions for the normalized standard error of sample estimates of the mean, variance, and covariance of the streamwise and vertical velocities, expressed in terms of the Eulerian integral time scale. He also suggests that at least 30 bursts per record should be considered to get reliable statistics of the turbulent quantities. Nezu and Nakagawa (1993), based on Taylor's hypothesis and on the turbulence macroscale, proposed a criterion to determine the maximum response frequency of a measuring instrument that would allow one to analyze the spectral distribution down to the viscous subrange. They suggest that the dimensionless wave number should be at least equal to 100 to be able to perform such an analysis. They also suggest that a record containing at least 100 burst events should be used to examine the turbulent structures averaged over the long term.

To the authors' knowledge the error for estimating mean velocities due to the sampling time span of ADCP measurements has never been assessed using the integral time scale. In the statistical description of stationary turbulent flows, the 
integral scale is assumed finite. This scale is a rough measure of the time interval over which a fluctuating quantity is correlated with itself and, at the same time, a time measure over which the quantity is dependent on its past values (Tennekes and Lumley, 1972). In this paper we present an analysis aimed at evaluating the error of ADCP measurements due to the sampling time span based on the integral scale. The integral time scale at different depths is estimated based on the autocorrelation function of the velocity record and is used to evaluate the mean-square error of average velocities as a function of the integration time. The implications of these errors in typical ADCP measurements for discharge estimates in natural streams are discussed.

\section{Field measurements}

For over five years the U.S. Geological Survey (USGS) has been measuring flows in the main branch of the Chicago River at Columbus Drive as part of the Lake Michigan Diversion Accounting Program. Flows need to be estimated at this site by the USGS to monitor diversion from Lake Michigan by the State of Illinois. The Chicago River at Columbus drive has a cross section approximately rectangular about $200 \mathrm{ft}$ wide and $6 \mathrm{~m}$ deep. A 30-minute segment of an 18-hour velocity record collected in the central region of the river at this station on September 17, 1998 between 23h00s and 23h30s is used for the analysis presented herein. This velocity record consists of single-ping data measured at a sampling frequency of $1 \mathrm{~Hz}$ with an uplooking broadband ADCP operating at $600 \mathrm{kHz}$. These data belong to a set of measurements undertaken to monitor the streamwise velocity during low flow periods in the river. Data were collected using bin size of $0.25 \mathrm{~m}$. Due to the blanking distance and the distance from the transducers to the center of the bin, the first measured bin was at approximately $1.1 \mathrm{~m}$ from the channel bed. The velocity record is expected to approximately have $2 \mathrm{D}$ properties because it was collected in the central region of the channel, i.e., outside the region where secondary currents due to sidewall effects are important (Nezu and Rodi, 1985). Moreover, because ADCP's are relatively accurate for measuring velocities in 2D flow fields such as ocean currents and flow through wide open channels, it is reasonable to assume that the spatial averaging performed by the ADCP only has a mild effect on the accuracy of the data.

\section{Data analysis}

The four 30-min sets of ADCP velocity measurements used in the analysis presented here are shown in Fig. 1. These measurements represent the velocity time series at 1.1, 2.1,3.1, and $4.1 \mathrm{~m}$ from the riverbed collected at sampling intervals of 1 second. The stationarity of the data was verified with the run test (Brendat \& Piersol, 1985) following the approach used by Soulsby (1980). More specifically, the stationarity of the mean velocities and turbulence intensities was tested by applying the run test to a series of the average values of these parameters obtained by using 30 records of 1-minute duration generated by further dividing each of the data sets. The results of the run test are shown in Table 1. The range of runs for acceptance of the hypothesis of independence, and conse- 
quently of stationarity, at the level of 0.05 is between 10 and 21 . Results in Table 1 indicate that both the average and variance of the streamwise velocities were stationary throughout the time span of the 30 -min record.

The integral time scale $\mathcal{T}$ of a fluctuating quantity is defined as the integral of the respective autocorrelation function (ACF) between a lag time $\tau=0$ and $\tau \rightarrow \infty$. This scale, as suggested by Tennekes and Lumley (1972), can be used to assess the convergence of averages. Thus, the mean-square error of an estimate of the mean velocity based on a record of measurements of finite length $T$ much longer than the integral time scale $\mathcal{T}$, can be approximated as:

$$
\overline{\left(U_{T}-U\right)^{2}} \approx 2 \overline{u^{2}} \mathcal{T} / T
$$

in which:

$$
\begin{aligned}
U & =\text { ensemble mean velocity; } \\
U_{T} & =\text { mean velocity estimate based on a record of length; } \\
\mathbf{u}^{2} & =\text { variance of streamwise velocity; }
\end{aligned}
$$

The mean-square error normalized with the variance based on this relation is plotted in Figure 2. As shown by Figure 2, estimates of the mean velocity based on a record of length equal to $10 \mathcal{T}$ will approximately have a mean-square error equal to 0.2 the ensemble variance. Conversely, as indicated by the expression above, to achieve a mean-square error equal to 0.01 the ensemble variance, a record of length $200 \mathcal{T}$ is necessary.

In the present analysis of ADCP data, the ACF is estimated via direct computations (Bendat and Piersol, 1986). The integral time scale, on the other hand, is estimated by integrating the ACF between $\tau=0$ and the time lag corresponding to the first zero-crossing of the ACF. The estimates of the mean, variance and integral time scale of the streamwise velocities obtained with the 30-min data records are shown in Table 2. The estimates of the integral time scale are between 20 and $28 \mathrm{~s}$, showing an inverse dependence on the distance to the channel bed. The mean-square errors of the mean velocity normalized with the ensemble variance estimated using two different values of the $T$ are also listed in Table 2. Noticeably, the mean velocity errors of the 5-minute estimates would be between 0.13 and 0.18 the ensemble variance, depending on $\mathrm{z} / \mathrm{H}$. Conversely, the mean-square error of the velocity estimates would be between 0.02 and 0.03 the ensemble variance when the 30-min record is used.

An alternative approach that has been used to determine the record length needed to estimate mean velocities makes use of a plot of the mean velocity as a function of the averaging time span (e.g., González et al., 1996). This approach has also been used to determine the averaging time necessary to estimate turbulence quantities (e.g., Barua, and Rahman (1998). The differences between this approach and the one described above are contrasted below.

The mean-square errors of the mean velocity estimates as a function of the averaging time with respect to the mean velocity of the whole data record and 
normalized with the standard deviation for $\mathrm{z} / \mathrm{H}=0.71$ are shown in Figure 3 . The plot shown in Figure 3 suggests that if a data record of 60 seconds were used, the mean velocity would be estimated with a normalized mean-square error equal to 0.12 . Conversely, for the same record length of 60 seconds, and given that $\mathcal{T}=20$ seconds (see Table 2 ), the mean velocity would be estimated from Figure 2 with a normalized mean-square error equal to 0.66 . The normalized mean-square errors shown in Figure 2 are expressed in terms of the ensemble mean and variance, while the mean-square errors in Figure 3 are expressed in terms of the long-term mean and variance. Therefore, the plot in Figure 3 is expected to be a better measure of the mean-square error of the average velocity than the plot in Figure 2.

\section{Conclusions}

As discussed above, use of the integral time scale provides an objective means to assess the averaging error as a function of the record length. The length of the sampling time necessary to estimate the streamwise mean velocity within a prescribed accuracy in terms of the mean-square error is clearly a function of the integral time scale. The integral time scale can thus be used to either determine the sampling time necessary to achieve a prescribed accuracy of the mean velocity or to determine the mean-square error of velocity estimates expected from a record of a given time length.

In practical applications the integral time scale needs to be estimated first. This, as explained in the former section, can be approximately evaluated by integrating the ACF between zero and the first zero-crossing lag time. On the other hand, the accuracy of the estimates of the autocorrelation coefficients is inversely proportional to their value (Bendat and Piersol,1986); therefore, to obtain reliable estimates of the integral time scale the ACF should be evaluated using relatively long records.

It is worth noting that for situations where the ACF and the integral time scale cannot be determined, a rough estimate of the integral time scale can be obtained by dividing the streamwise integral macroscale of the turbulence, $L_{x}$, by a first-order estimate of the mean velocity. The streamwise integral macroscale of turbulence can in turn be estimated with a semi-theoretical relationship such as that proposed by Nezu and Nakagawa (1983) given by $L_{x} / H=(z / H)^{2}$ for $z / H \leq$ 0.6 , and $L_{x} / H=0.66$ for $z / H>0.6$, where as in the previous section $z$ is the distance from the channel bed and $\mathrm{H}$ is the flow depth. This approach is an approximation only valid for flow conditions in which Taylor's hypothesis holds.

The analysis presented herein suggests that the accuracy of streamwise mean velocities based on fixed ADCP measurements are affected by the temporal resolution of the data. Results show that there is a clear dependence of the mean velocity estimates on the averaging time, and consequently of discharge estimates as well. Furthermore, the discharge through the top/bottom parts of the profiles where ADCPs cannot measure is usually estimated by the ADCP based on extrapolations of the measured data (Gordon, 1995). However, although streamwise velocity profiles can be extrapolated based on the power law, this extrapolation is strictly applicable only to long-term profiles in fully developed 
boundary layers, which in turn can only be accurately determined based on long records. Therefore, the effect on the accuracy of the discharge estimates of the commonly used approach consisting of extrapolating velocity profiles estimated with only a few pings needs to be carefully assessed. A desirable test to assess the effect of the temporal resolution on discharge estimates would be to compare discharge estimates based on profiles with a prescribed mean-square error collected with a fixed ADCP at different spanwise locations in a river with typical discharge estimates based on ADCP transects.

The accuracy of other flow quantities such as turbulence intensities and Reynolds stresses is not only more dependents upon the record length than the average velocities, but as described in the first section of the paper, it also depends upon the sampling frequency. Soulsby (1980) suggests that due to flow intermittence and its effect on the inherent variability of the Reynolds stress, reliable estimates of this quantity can only be obtained if a record containing no less than 30 bursts is used. Nezu and Nakagawa (1993) indicate that the ratio of the product of the mean bursting period times the mean bulk streamwise velocity and the flow depth is between 1.4 and 2.7. They also recommend that a record containing at least 100 burst events should be used if the long-term turbulent structures of the flow are to be analyzed.

\section{References}

Barua, D. K. and Rahman, K. H. (1998), "Some Aspects of Turbulent Flow Structure in Large Alluvial Rivers," J. Hydr. Res., 36(2), 235-252.

Bendat, J.S., and Piersol, A. G. (1986). Random Data: Analysis and Measurement Procedures. Wiley-Interscience.

Droz, C. J., López, F. y Prendes, H. (1998) "Mediciones de Velocidades y Caudales con un ADCP en el Río Paraná." XVII Congreso Nacional del Agua y II Simposio de Recursos Hídricos del Cono Sur, Santa Fé, Argentina. Aug. 1998. (In Spanish)

González, J. A., Melching, C.S., and Oberg, K. A. (1996) "Analysis of OpenChannel Velocity Measurements Collected with an Acoustic Doppler Current Profiler," Proc. RiverTech96, IWRA, Chicago, Illinois. Vol. 2, 838-845.

Gordon, R. L. (1996), Acoustic Doppler Current Profilers. Principles of Operation: A Practical Primer. Second Edition for Broadband ADCPs. RD Instruments.

Gordon, R. L. (1989), "Acoustic Measurement of River Discharge," J. Hydr. Engrg., ASCE, 115(7), 925-936.

McQuivey, R. S. (1973). "Summary of Turbulence Data from Rivers, Conveyance Channels and Laboratory Flumes," Prof. Paper 802-B. U.S. Geological Survey,

Nezu I. y Nakagawa H. (1993). Turbulence in Open-Channel Flow. IAHR Monograph Series. A.A. Balkema, Rotterdam.

Nezu, I., and Rodi, W. (1985). "Experimental study on secondary currents in open channel flow," Proc., $21^{\text {st }}$ Congress of IAHR, Melbourne, Australia, Vol. 2, 115-119. 
Tennekes, H. and Lumley, J. L. (1972). A First Course in Turbulence. MIT Press. Cambridge, Massachusets.

Soulsby, R.L. (1980). "Selecting record length and digitization rate for near-bed turbulence measurements". J.Phys. Ocean. 10, 208-219.

Table 1. Number of runs for run test on 1-min estimates of mean and turbulence intensities of streamwise velocities of data sets.

\begin{tabular}{ccc} 
& \multicolumn{2}{c}{ Runs } \\
\cline { 2 - 3 } Z/h & $\bar{U}$ & $\overline{u^{2}}$ \\
0.18 & 15 & 12 \\
0.35 & 15 & 10 \\
0.52 & 13 & 13 \\
0.68 & 14 & 15
\end{tabular}

Table 2. Estimates of mean, variance, integral time scale, and normalized meansquare error of the streamwise velocities from ADCP single-ping 30-min records collected in the central region of the Chicago River at Columbus Drive.

$\begin{array}{lccccc}\mathbf{Z} / \mathbf{h} & \bar{U} & \overline{u^{2}} & \mathcal{T} & \frac{\overline{\left(\mathrm{U}_{\mathrm{T}}-\mathrm{U}\right)^{2}}}{\overline{\mathrm{u}^{2}}} & {[\%} \\ & {[\mathrm{cm} / \mathrm{s}]} & {[\mathrm{cm} / \mathrm{s}]^{2}} & {[\mathrm{~s}]} & \mathrm{T}=5 \mathrm{~min} & \mathrm{~T}=30 \mathrm{~min} \\ & 3.6 & 1.3 & 20 & 13.3 & 2.2 \\ 0.71 & 3.5 & 1.2 & 23 & 15.3 & 2.6 \\ 0.55 & 3.1 & 1.2 & 28 & 18.7 & 3.1 \\ 0.39 & 2.5 & 1.2 & 26 & 18.0 & 3.0 \\ 0.22 & & & & \end{array}$




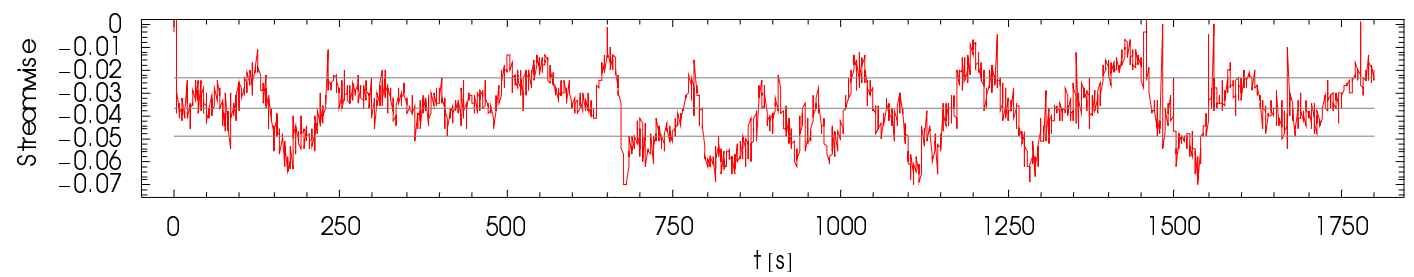

(a) $\mathrm{z} / \mathrm{H}=0.71$.

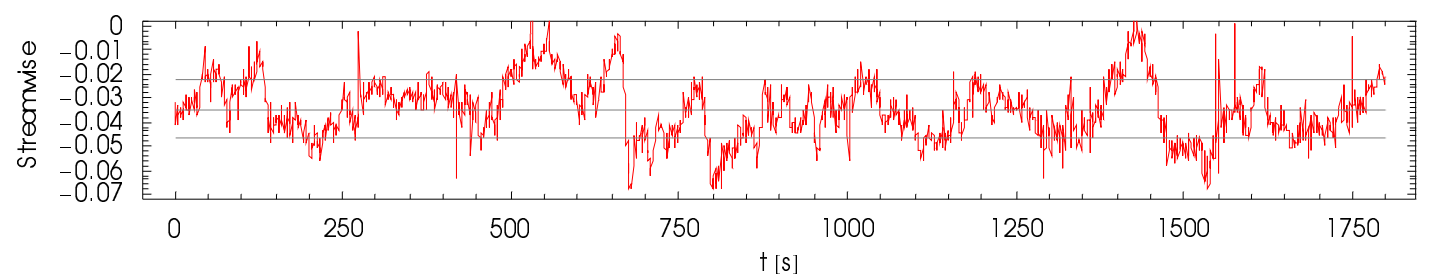

(b) $\mathrm{z} / \mathrm{H}=0.55$.

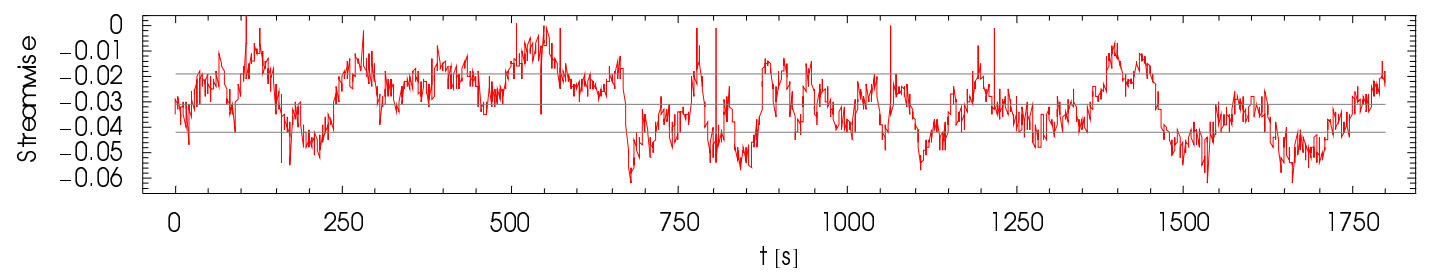

(c) $\mathrm{z} / \mathrm{H}=0.39$.

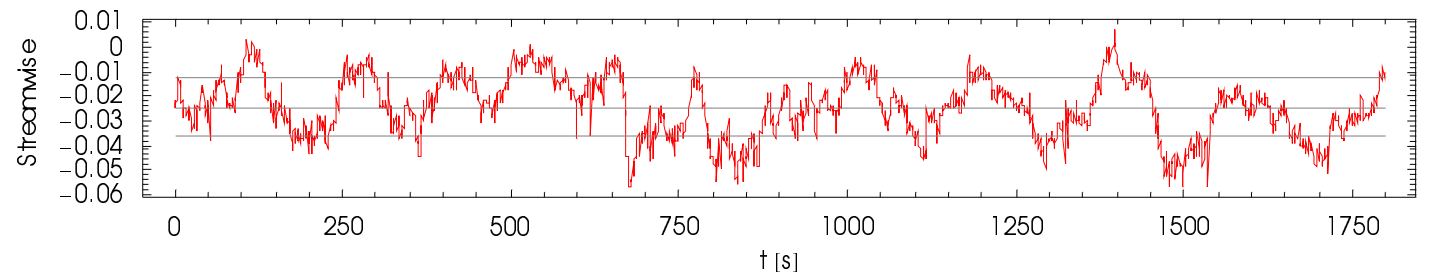

(d) $\mathrm{z} / \mathrm{H}=0.22$.

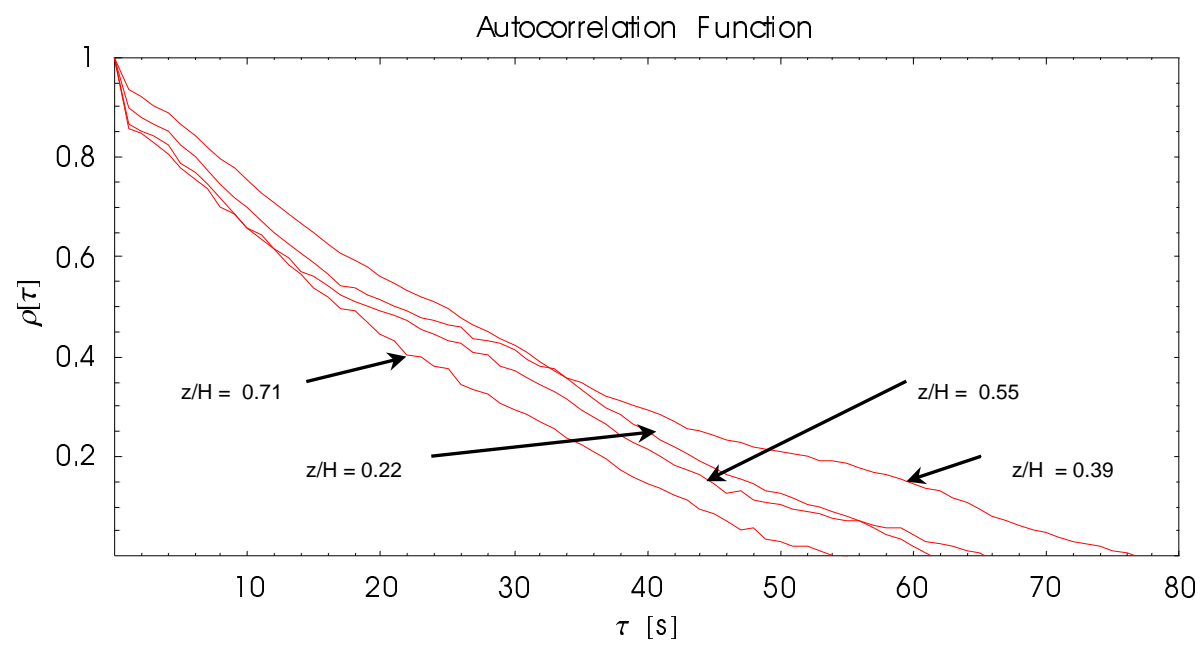

Fig. 1 Streamwise velocities measured with an ADCP at different distances from the bed in the Chicago River at Columbus Drive and respective ACFs. 


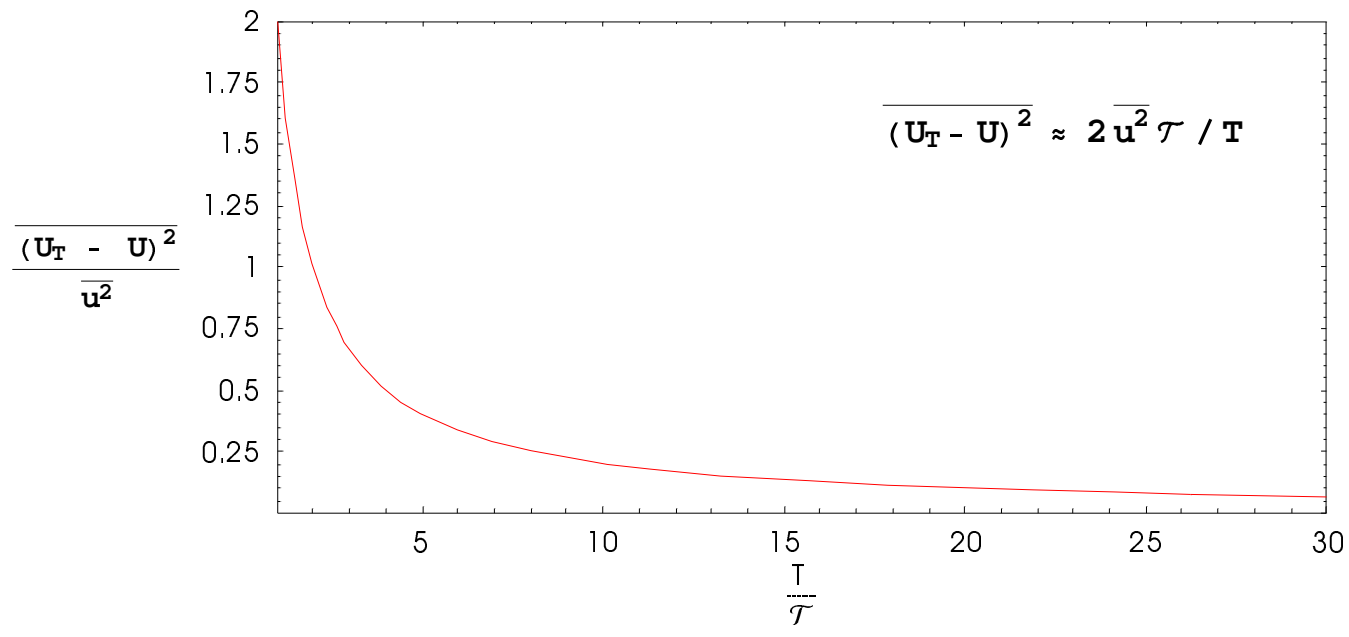

Fig. 2 Normalized mean-square error of mean velocity estimates as a function of record length expressed in terms of the integral time scale.

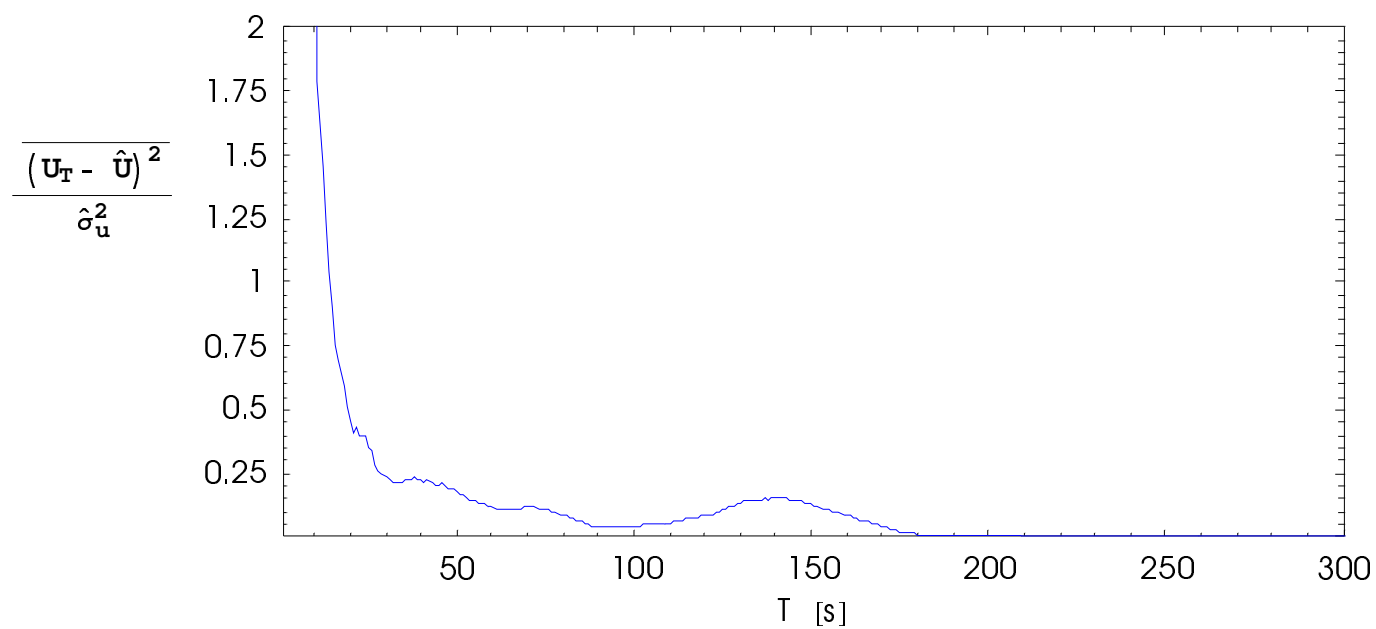

Fig. 3 Normalized mean-square error of mean velocity estimates expressed in terms of long-term mean and variance as a function of record length for the velocity time series measured at $\mathrm{z} / \mathrm{H}=0.71$. 